\title{
Questes
}

\section{Aux marges de la nuit. Le voyage de l'âme dans l'au-delà et la symbolique du passage}

\section{Mattia Cavagna}

\section{(2) OpenEdition}

\section{Journals}

\section{Édition électronique}

URL : http://journals.openedition.org/questes/1955

DOI : 10.4000/questes. 1955

ISSN : 2109-9472

\section{Éditeur}

Les Amis de Questes

\section{Édition imprimée}

Date de publication : 15 janvier 2004

Pagination : $30-34$

ISSN : 2102-7188

\section{Référence électronique}

Mattia Cavagna, « Aux marges de la nuit. Le voyage de l'âme dans l'au-delà et la symbolique du

passage », Questes [En ligne], 6 | 2004, mis en ligne le 01 janvier 2014, consulté le 15 septembre 2020. URL : http://journals.openedition.org/questes/1955

\section{(c) Association des amis de «Questes »}


du chevalier Owein, il nous paraît plus malaisé de parler de la nuit car nous ne voulons pas la confondre avec l'obscurité. De plus, la temporalité de l'autre monde ne nous paraît pas pouvoir s'analyser dans les termes de nuit ou de jour, comme celle du nôtre. Autre monde, autre temps !

\section{Aux marges de la nuit. \\ Le voyage de l'âme dans l'au-delà et la symbolique du passage}

\section{Mattia CAVAGNA}

Dans l'imaginaire chrétien médiéval, la frontière entre la réalité terrestre et l'audelà est conçue comme une limite tout à fait perméable qui peut être franchie dans l'un ou l'autre sens. La nuit contribue ultérieurement à nuancer cette frontière : l'obscurité favorise les différents types de contacts entre les deux mondes, à savoir les apparitions des saints et des revenants, mais surtout les rêves et les voyages extatiques des hommes.

Entre ces deux dernières catégories de visions il existe des différences importantes qui se rapportent justement à la dimension nocturne. Si d'un côté les rêves comportent le sommeil du protagoniste, de l'autre les voyages dans l'au-delà impliquent, au contraire, un état de veille qui se traduit en un processus tout à fait dynamique : la séparation de l'âme du corps et son départ dans l'audelà. Nous voudrions concentrer notre analyse sur ce type d'expérience 
extatique qui, selon les textes de notre corpus, s'effectue toujours aux marges de la nuit : soit au coucher du soleil (le plus souvent) soit à l'aurore.

Pour proposer quelques exemples, nous citerons d'abord la Vision de Drithelm, un texte relaté par Bède le Vénérable $\left(\mathrm{VIII}^{\mathrm{e}}\right.$ siècle) : le voyage extatique est ici situé explicitement au moment de la «première partie de la nuit » (primo tempore noctis) ${ }^{31}$. De même, la Vision de Jehan de Lüttich (1147) a lieu pendant que les moines ses frères sont occupés avec «l'office des vêpres » (vespertinae laudis officio $)^{32}$. Un troisième exemple, que nous voudrions évoquer plus en détail, est constitué par la Vision de Charles le Gros (IX ${ }^{\mathrm{e}}$ siècle). Après la messe du soir, l'empereur part se coucher et, au moment où il essaye de s'endormir, surgit une voix qui lui annonce son voyage imminent dans l'au-delà :

sacra nocte Dominici diei post celebratum nocturnarum Horarum divinum officium, dum vellem carpere somnum, venit vox ad me terribiliter dicens : "Carole, exiet modo a te spiritus tuus $(\ldots) »^{33}$

Nous voudrions souligner ici cette expression «dum vellem carpere somnum » : l'empereur manifeste sa volonté de dormir, mais la voix surnaturelle l'en empêche.

En effet, d'après nos sources, le sommeil est conçu comme une condition statique qui s'oppose à la dynamique de l'extase. Il constitue en quelque sorte un élément de frein. Cela est évident dans certains textes où le protagoniste du voyage se trouve entouré par d'autres moines qui dorment profondément autour

\footnotetext{
31 Bède le Vénérable, Historia Ecclesiastica Gentis Anglorum, éd. B. Colgrave et R.A.B. Mynors, Oxford, 1991 p. 488.

32 Visio cujusdam monachi de statu animarum post mortem, éd. Migne, PL 180, col. 177.

33 Visio Caroli Grossi, éd. Migne, PL 174, col.1287.
} 
de son lit. C'est le cas, par exemple, de la Vision de Wetty (IX ${ }^{\mathrm{e}}$ siècle) mais surtout de la Vision du moine Auguste (début du VII ${ }^{\mathrm{e}}$ siècle) qui met en scène une situation très significative. Le moine Auguste est malade et, puisqu'il est très jeune, il reçoit quotidiennement un grand nombre de visites de ses frères. Il n'est jamais laissé seul, et dans sa chambre il y a toujours un cierge allumé. Un soir, l'abbé, qui est l'auteur du récit, se rend dans la cellule du malade et découvre que tous les moines sont endormis autour de son lit. Ils dorment si profondément que personne ne s'aperçoit de son entrée. De plus le cierge qui normalement éclairait la chambre se trouve éteint :

Ingressusque cellam in qua ipse (..) recubabat, cunctos qui aderant ita repperi sopore depressos, ut nullus eorum ad meum fuisset expergefactus introitum. Lumen vero, quod ibi accensum erat, extinctum inveni ${ }^{34}$.

Ensuite, Auguste révèle qu'il a eu une vision magnifique et il décrit à tous ses frères le Paradis et l'Enfer qu'il a visités. Il ne spécifie pas s'il a été ravi en extase, mais il affirme qu'il n'a pas rêvé, car il n'a pas dormi du tout, alors que les autres étaient justement «gravés par le sommeil » (sopore depressos).

Avant de tirer quelques conclusions, nous voudrions citer un dernier exemple de vision qui a lieu au moment de l'aube. Il s'agit de la Vision de Baldarius $\left(\mathrm{VII}^{\mathrm{e}}\right.$ siècle). Dans ce cas aussi, le moine se trouve malade dans son lit; il raconte que son âme est partie de son corps et a été accueillie par trois colombes au moment où la nuit laissait la place à la lumière du jour :

\footnotetext{
34 De obitu Agusti, dans Liber de Vitas Patrum Emeretensium, éd. Joseph N. Garvin, The vitas Sanctorum patrum Emeretensium, the Catholic University of America Press, Washington D. C., 1946, p. 138.
} 
Cum autem, inquit, grave aegritudine oppressus jacerem exanimis praetereunte noctis spatio, exsurgente lucis crepusculo, anima mea egressa e corpore suscepta est a tribus splendidissimis columbis ${ }^{35}$

Le départ de l'âme s'effectue ici dans un moment précis : celui du passage entre l' «espace de la nuit » et l'arrivée de la lumière matinale. Il faut ici souligner la suggestive opposition entre les deux participes présents «pretereunte » et «exsurgente $»$ : il s'agit de deux verbes (praeter-eo et ex-surgo) qui évoquent justement l'idée d'une action, d'un mouvement, d'une mutation en acte. Les dimensions de l'espace et du temps se trouvent également mises en enjeux : l'expression «noctis spatio » suggère justement la correspondance entre les deux plans. Au passage entre la nuit et le jour correspond le passage de l'âme du visionnaire : elle quitte la dimension terrestre pour rentrer dans l'espace de l'éternité.

Dans le dernier exemple cité, la valeur symbolique de ce passage est particulièrement explicite : l'image de l'aube, de la lumière du soleil qui surgit est évidemment un signe positif. Le voyageur sera conduit par les trois colombes-anges en direction de l'Orient, (partem Orientis) qui est naturellement, associé au Paradis ${ }^{36}$.

C'est donc sous cette perspective qu'il faut interpréter le choix de situer les visions aux marges de la nuit. Il s'agit d'une symbolique profonde, liée au moment du passage entre le jour et la nuit, entre les ténèbres et la lumière (ou

\footnotetext{
35 Valerius du Bierzo, De coelesti revelatione, éd. Migne PL 87, coll. 435

36 Le concept d' «Orient» (<du lat. orior = surgir) participe également de cette symbolique liée à l'image du soleil et de la lumière matinale.
} 
vice-versa). La vision se configure effectivement avant tout comme un passage d'un état à l'autre, d'une condition terrestre, corporelle, à une condition extatique ; un passage qui présente un caractère tout à fait actif et dynamique.

Pour envisager une enquête plus complète et approfondie, il serait souhaitable de considérer également le domaine des rêves, dont le corpus a des dimensions très importantes. A ce propos, nous citerons seulement un texte du $\mathrm{XI}^{\mathrm{e}}$ siècle, la Vision de Bernhard, évoquant un rêve qui a lieu « après minuit »:

Post medium noctis cum pulso torpore somnia sunt verissima, videbar sub divo cum aliis quatuor stare ${ }^{37}$

L'auteur souligne que l'expérience a lieu «post medium noctis», lorsque le sommeil est plus profond: cela semble garantir la véridicité des rêves. Il apparaît de toute évidence qu'il s'agit d'une approche très différente des récits que nous venons d'analyser. Le choix de situer le rêve au milieu de la nuit correspond à une logique opposée à celle des voyages extatiques : le rêve est, par définition, une opération contemplative et donc statique. Le milieu de la nuit et le sommeil profond sont alors les circonstances idéales du rêve. Cela s'oppose justement à la condition active et dynamique de l'âme en extase, une âme qui préfère, pour son départ, le moment des vêpres ou du soleil levant, un peu comme Claude Monet.

37 Visio Berhardi, éd. F.J. Mone, Quellensammlung der Badischen Landesgeschichte, Karlsruhe, 1848, p 23. 